\section{IMPROVING MOUTH COMFORT}

Ortho-Care Ltd, one of the UK's largest independent manufacturers and distributors of orthodontic products, is introducing ComfortBrace to the UK. A much more effective alternative to dental wax, ComfortBrace protects the mouth from the wear and tear of metal braces.

The brand new product, which has been developed in the US, is already proving to be very popular with fixed lingual brace wearers. The clear, thin plastic protective strips which adhere to teeth and braces significantly improve mouth comfort by eliminating pain and irritation.

Wearers simply use scissors to cut the strip to size, peel off the backing, moisten their teeth with saliva or water and apply across their braces wrapping securely around brackets and wires. The advanced adhesive technology ensures that the strips stay firmly in place forming a protective layer between the brace and lips.

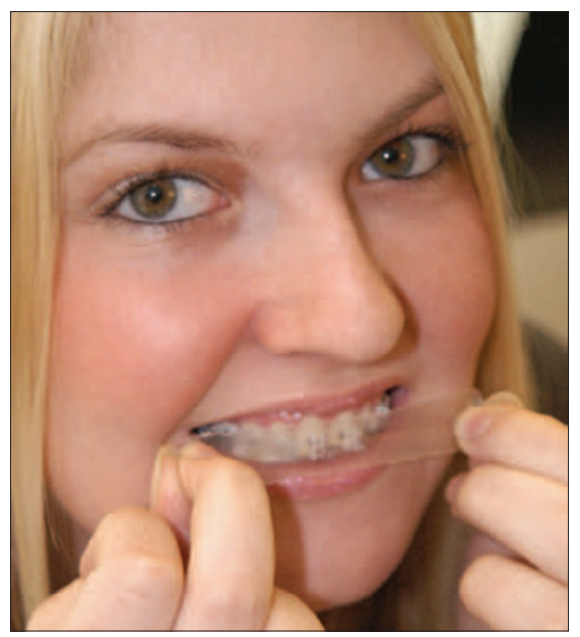

ComfortBrace testers say the strips are far superior to dental wax because they stay in place, there is no need to reapply protection and ComfortBrace doesn't melt when enjoying hot foods and drinks. It is also really easy to remove, peeling off in one piece, with any adhesive residue simply washing off when brushing teeth.

ComfortBrace retails at $£ 19.50$ for a box of 24 strips.

Reader response number 57

\section{AWARD-WINNING ORAL CARE FOR INFANTS}

Leading child oral health company Brush-Baby is celebrating success after its founder Dominique Tillen was awarded an Entrepreneur of the Year award for her pioneering and innovative work launching

Brush-Baby.

Brush-Baby's concept of an 'oral care pathway for babies and young children', is built around a highly original, contemporary product range whose ultimate aim is to 'improve the oral health of young children'. The company's first product, the BrushBaby Chewable Toothbrush, was launched in 2009. The first of its kind worldwide, it is a combined versatile chewable toothbrush and teether that toddlers can use themselves, satisfying their natural urge to chew. As they do so, it helps to clean teeth, massage gums and soothe tender teething gums.

The complete Brush-Baby range includes DentalWipes, innovative

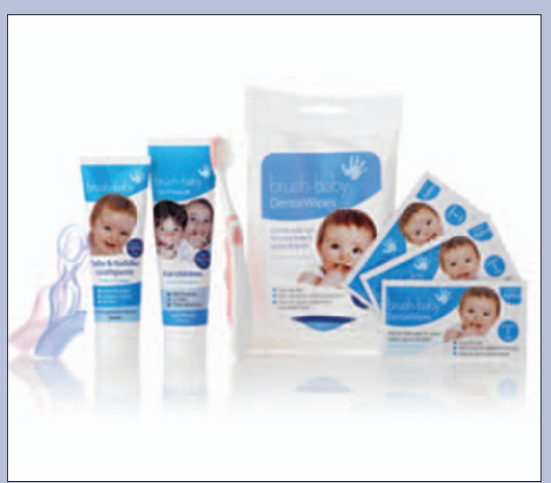

toothbrushes and xylitol/fluoride toothpastes for babies and children aged from birth to six years.

Eye-catching packaging is backed by stand-out POS graphics and engaging pack photography, and offers significant cross-selling opportunities to further energise consumer demand. The product packaging has also been cleverly designed to work with shelf spacesaving merchandising.

Reader response number 58

\section{SAVE TIME ON MIXING}

Self-adhesive luting cement G-CEM from GC UK is now available in an automix presentation. The new dual-chamber cartridge saves time on mixing and applying the product, while the innovative chemical formula with specific adhesive monomers ensures that dental restorations are more securely fitted and will last longer.

G-CEM is a dual-cure self-adhesive universal resin cement designed for adhesive luting of all-ceramic, metal or composite indirect restorations. With CAD-CAM and metal-free restorations becoming so popular, G-CEM was developed with the aim of combining improved handling and self-adhesion of conventional cements with the superior mechanical properties, adhesion and aesthetic qualities of resin cements.

Ensuring a high bond-strength to enamel in both self-cure and light-cure modes, G-CEM guarantees perfect bonding every time. There is no pre-conditioning on either the tooth or prosthesis and with a high fluoride release, secondary caries is no longer a concern.

Reader response number 59

\section{SUPERB SUPER BUFFER}

Shofu are well known for their comprehensive range of finishing and polishing instruments which include the SuperSnap range. As the perfect adjunct, Shofu have introduced the Super Buff paste-impregnated felt disk. The disk is simply moistened with water or the patient's saliva to release the unique polishing paste that helps to achieve an outstanding finish to your composites.

Super Buff is the first polisher on the market with a paste-impregnated disc. You do not have the mess of a syringe, and the polishing material will not splash you or the patient. Super Buff is easily activated by water or saliva, and it works with all composites on multiple surfaces. Above all the final finish is superb.

Reader response number 60 